\title{
Pediatric haematopoiesis and related malignancies (Review)
}

\author{
MINGWEI JIN*, SHUMEI XU* and QI AN \\ Department of Pediatrics, Xuzhou Children's Hospital, Xuzhou, Jiangsu 221002, P.R. China
}

Received January 25, 2017; Accepted April 18, 2017

DOI: $10.3892 / \mathrm{ol} .2017 .6106$

\begin{abstract}
Survival after acute paediatric (0-14 years), adolescent (15-19 years) and young adult (20-39 years) leukaemia has improved substantially over the last five decades, particularly for acute lymphoblastic leukaemia (ALL) and acute promyelocytic leukaemia. This progress represents one of the most successful achievements in the history of medicine and has been attributed to the development of effective chemotherapy regimens, improvement in supportive care, better risk stratification, use of targeted therapies, and advances in haematopoietic stem cell transplantation. Recent studies have revealed improvement in survival over time for all age groups and subtypes of leukaemia. However, these outcomes varied widely by age and are associated with sociodemographic and clinical factors. The present review concludes that survival and early death after acute leukaemia has greatly improved among young patients. However, inequalities in outcomes remain and are likely a result of multiple factors.
\end{abstract}

\section{Contents}

1. Introduction

2. Diagnosis and classification

3. Clinical features

4. Risk stratification, treatment and prognosis

5. Acute lymphoblastic leukaemia

6. Adolescent and young adult acute lymphoblastic leukaemia

7. Acute myeloid leukaemia

8. Core-binding factor AML

9. Down syndrome effects

10. Neonatal and infant acute myeloid leukaemia

11. Conclusion

Correspondence to: Dr Qi An, Department of Pediatrics, Xuzhou Children's Hospital, 18 Sudibei Road, Xuzhou, Jiangsu 221002, P.R. China

E-mail: aiqiangel@yeah.net

*Contributed equally

Key words: pediatric malignancies, leukemia, myeloid leukemia, haematopoiesis

\section{Introduction}

Haematopoiesis relates to the production, proliferation, self-renewal, and differentiation of blood cells (1). In response to growth factors such as stem cell factor glycoproteins (interleukins 1 to 7) and colony-stimulating factors, multipotent haematopoietic stem cells generate and maintain all differentiated lymphoid and myeloid cells present in the blood, bone marrow, spleen, and thymus (2). The haematopoietic stem cells produce two progenitor cells: the common myeloid and common lymphoid cells. The common lymphoid progenitor cell originates the natural killer, T- and B-cells that are part of the immune system and have the key role of controlling infections. The common myeloid progenitor cell generates three lineages of cells: erythrocyte, megakaryocyte, and myeloblast. The erythrocyte (red blood cell) is responsible for carrying and delivering oxygen to the body organs and tissues. The megakaryocyte produces the platelets or thrombocytes, responsible for blood clotting. The myeloblast cell differentiates into four types of cells, which have the capability of defending the body against infection and toxins: neutrophils, eosinophils, basophils and monocytes. Fig. 1 shows a schematic representation of human haematopoiesis.

Leukaemic transformation of a progenitor haematopoietic cell involves a disruption in the course of normal proliferation and differentiation process, resistance to apoptotic signals, and increased self-renewal. The prevalent theory of leukaemogenesis is that a single haematopoietic cell suffers mutation and goes into an unlimited process of self-renewal resulting in malignant, poorly differentiated haematopoietic cells (clonal origin of leukaemic cell) (3). Leukaemia cells behave differently than normal haematopoietic precursors, with slower cell division and longer time to produce DNA. Yet, these cells accumulate persistently in the bone marrow of leukaemic patients and progressively replace haematopoietic cells. Furthermore, this causes bone marrow failure that is associated with severe anemia, bleeding, and infections.

\section{Diagnosis and classification}

Previously, morphologic analysis was used to classify leukaemia into myeloid or lymphoid (4). Currently, advanced diagnostic techniques such as flow cytometry, conventional and molecular genetics as well as next-generation sequencingbased multi-gene mutation profiling provide precise diagnosis and classification of leukaemias. Some tests are useful not only for diagnosis, but also to evaluate if therapy has been 


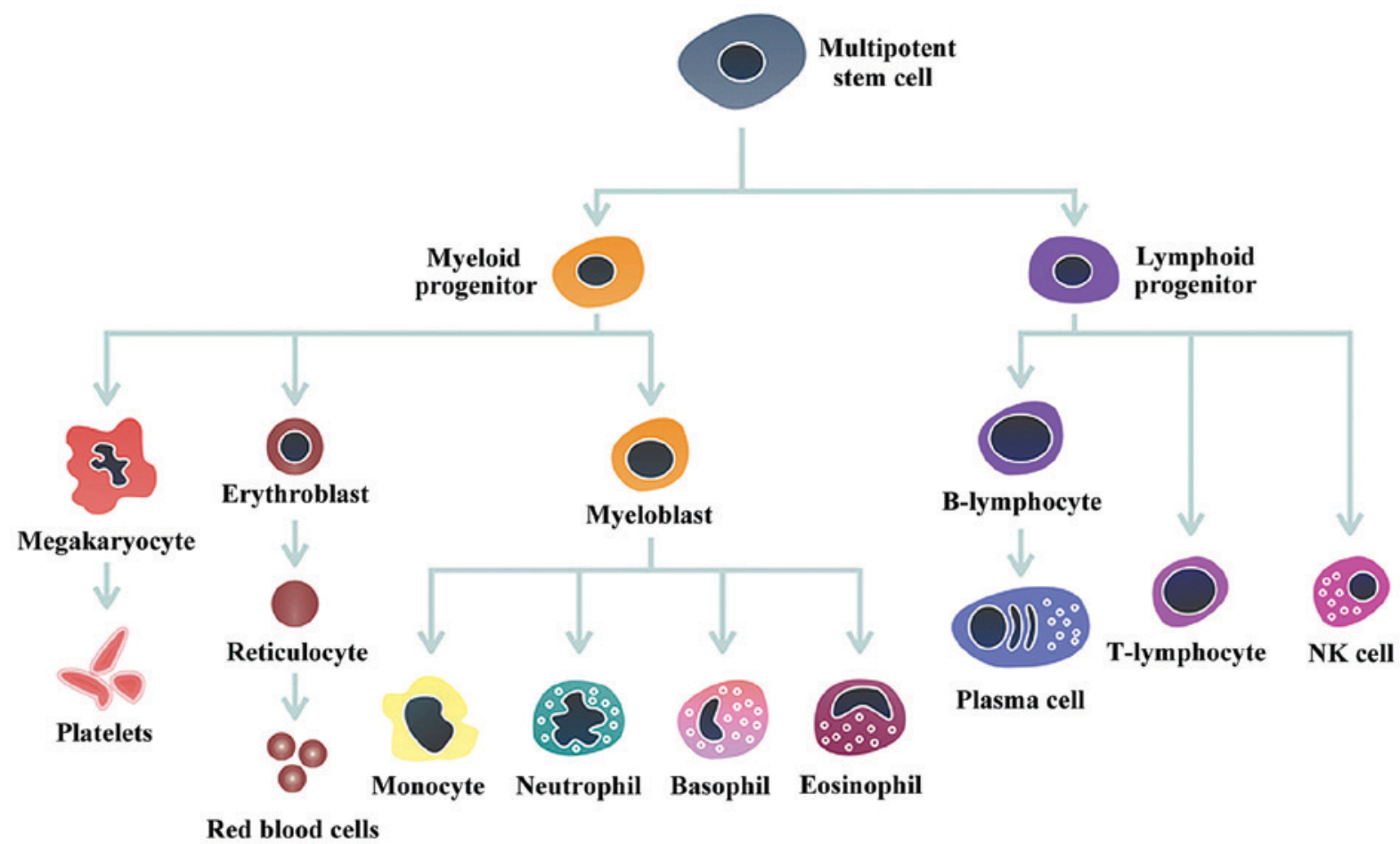

Figure 1. Haematopoiesis in humans.

effective or modifications of the initial treatment is required. The common tests used to determine initial response to therapy include the fluorescence in situ hybridization test, flow cytometry and polymerase chain reaction (PCR) (5). The diagnosis of leukaemia often performed by analysis of the peripheral blood. However, bone marrow examination is also required because up to $20 \%$ of patients might not present with blasts in the peripheral blood at the time of clinical presentation (6). In addition, the morphology of leukaemia cells in the blood might differ from the cells in the bone marrow.

\section{Clinical features}

The clinical features of acute leukaemia are secondary to the accumulation of malignant cells with consequent bone marrow failure. Symptoms observed or recorded are often non-specific. These include, easy or spontaneous bruising, fever, lethargy, pallor and infection. Bone pain and/or limping are more common symptoms. Physical examination might also show lymphadenopathy, hepatomegaly and weight loss.

Childhood cancer is a rare disease. Yet, the numbers of affected children are on continuous rise as the confirmed cases are rapidly increasing worldwide (7). With current population growth and decrease in childhood mortality rates (mostly due to lower mortality from infectious diseases), the incidence of childhood cancer is expected to increase by $30 \%$ by 2020 (8). About $70 \%$ of new cases are predicted to occur in low- and middle-income countries, where more than $80 \%$ of paediatric cancer deaths currently occur (9).

Leukaemia is the most common type of childhood malignancy, representing a third of all paediatric cancers and about $10 \%$ of malignancies in adolescents in the developed world. Acute lymphoblastic leukaemia (ALL) accounts for approximately $75-80 \%$ of all childhood leukaemias with an annual incidence rate of approximately 4/100,000 persons per year in the United States and Europe (7).

Acute myeloid leukaemia (AML) comprises about 15-20\% of all paediatric leukaemias, with an annual incidence rate of $0.8 / 100,000$ persons per year in the United States. Of those diagnosed with this malignancy, approximately $33 \%$ are adolescents and 50\% are adults in the developed countries (10). There is also substantial geographical variation in the incidence of AML, with the highest incidence rates reported in China, and Japan, and among the Maori population in New Zealand intermediate rates are reported. Although AML is a neoplasm more frequent in older people, this disease can occur at any age and remains the leading cause of cancer deaths among patients aged $\leq 39$ years (11). In contrast to ALL, there is no evidence of a significant increase of incidence of AML among children, adolescents and young adults in the United States and Europe over the last few decades.

\section{Risk stratification, treatment and prognosis}

The recognition that ALL and AML are heterogeneous disease has guided risk-directed therapy aimed at improving survival as well as the quality of life (12). The identification of patients with high risk of relapse invites intensive treatment; hence excessive toxic effects among cases with low-risk disease are prevented (13). Cytogenetic and molecular characteristics as well as assessment of minimal residual disease have been substituting many conventional prognostic factors in both ALL and AML. The risk stratification of acute leukaemias and several risk adapted-treatments are described in the following sections (14). 


\section{Acute lymphoblastic leukaemia}

Specific treatment approaches for ALL might differ depending on the disease presentation, but they regularly include remission-induction, intensification or consolidation treatment, and maintenance therapy to eradicate leukaemia cells. Patients with ALL generally need long maintenance treatment in order to prevent relapse. The mechanism by which lower dose chemotherapy regimen eradicates the residual leukaemic cells is poorly understood. When continuation treatment was given during a shorter period of time (18 months or less) for children and adults with ALL, survival was lower than that of conventional treatment. While approximately $65 \%$ of young patients may be cured with only 12 months of chemotherapy, it is not possible to identify these cases with certainty and the current recommendation is to treat these patients for two years or more; common drugs used for the treatment of ALL include corticosteroids, asparaginase and vincristine (15).

Currently, early response to therapy is evaluated by measurement of residual disease at specific time points in the peripheral blood and bone marrow, respectively. Minimal residual disease detected by PCR or by flow cytometry, is recognized as an important prognostic factor for survival in patients with ALL. This suggested that continuous minimal residual disease monitoring might be useful to identify patients with high or intermediate risk of relapse (those with a somewhat slow early response to therapy) and guide therapy. However, clinicians should be aware that some patients with persistent minimal residual disease may be cured, while some patients with minimal residual disease negative (undetectable) at remission can still present leukaemia recurrence (16). This emphasises the fundamental role of maintenance treatment for patients with ALL. Treatment directed at the central nervous system is of key importance and should be initiated early in the course of treatment. Several factors are taken into account when selecting the intensity of therapy, such as risk of relapse and the quantity of leukaemic cells in the cerebral spinal fluid. It has been established that cranial irradiation could cause various acute and late adverse effects such as secondary malignancies, neurocognitive disorders and endocrinopathies. Consequently, in many centres, intrathecal and systemic chemotherapy have replaced cranial irradiation (17).

Induction failure is rare in children, and could be defined as the presence of leukaemic cells in the peripheral blood, bone marrow or extra medullary location after 4-6 weeks of induction therapy. The children with ALL who have induction failure are considered very high-risk patients and haematopoietic stem-cell transplantation is recommended (18). Further, a study in the recent past confirmed higher survival time in children who had T-cell leukaemia with transplantation in comparison with the children who had undergone chemotherapy with transplantation (19). Detailed information on adolescents and young adults is comparatively scarce because of the smaller number of incident cases in this age group, as well as lower enrolment of these patients in clinical trials compared with children and older adults (20). Consequently, adolescents are commonly examined together with children aged 10-15 years in paediatric trials or with patients aged 20-30 years in adult trials. There is evidence that substantial decline in survival is observed beyond 15 years of age at diagnosis (21). At present, evidence suggests that intensified treatment protocols might reduce or eliminate the influence of some prognostic factors, such as male, black race and Down syndrome, on survival (22).

Moreover, although children with Down syndrome have an increased risk of developing ALL and lower survival, outcomes are comparable in children with and without Down syndrome after adjustment for favourable and unfavourable cytogenetic lesions (23). Clinical trials have identified several factors predictive of outcomes after ALL (24). The few particular subgroups of high-risk patients with ALL that should be treated with a risk-adapted protocol are described below. Due to the extremely poor prognosis in the past, children Philadelphia chromosome-positive ALL are currently treated with intensive chemotherapy plus imatininb or desatinib, tyrosine kinase inhibitors. Haematopoietic stem cell transplantation might be recommended in case of relapse.

\section{Adolescent and young adult acute lymphoblastic leukaemia}

The adolescents and young adults with ALL have poorer outcomes compared with children. In the mid-2000s, collaborative trials began to treat these patients with paediatric protocols and several studies have shown excellent results (25). Between 2007 and 2012, a large prospective adult intergroup trial (C10403) (26) in the United States, investigated the adoption of a successful protocol used by the Children's Oncology Group (ALL0232) for treatment of patients aged 16-39 years with ALL (27). The significant improvement in outcomes of B- or T-cell ALL patients supported the use of paediatric protocols by adult haematologists to treat adolescents and young adults with this neoplasm. However, despite improvement, there is evidence that these patients continue to be treated with low-intensity chemotherapy regimens in many centres (28). An explanation might be that some clinicians are not yet convinced about the paediatric approach superiority compared to the conventional treatment and may await more evidence from a randomized phase III study. Another possibility is that adult haematologists might not feel as familiar as paediatricians in managing the treatment-related toxicity secondary to the intensive paediatric protocol. Some complications of treatment such as pancreatitis, osteonecrosis, hyperglycemia, and infection seem to occur more often in older patients ( $>10$ years old). This might cause adult clinicians to change prescribed drug dosage and schedule $(29,30)$.

The observation of ALL relapse leading to deaths of pediatric patients still raises the risk of death in spite of the dramatic improvement in survival. The main determinants of survival are time to relapse, site of relapse, leukaemia immunophenotype, and more recently, minimal residual disease. Patients who present relapse within 36 months of diagnosis have a dismal 5-year overall survival of approximately $15 \%$ (31).

Salvage chemotherapy or even haematopoietic stem cell transplantation for both adults and children with relapsed or refractory ALL have not improved outcome, and intensive research continues to be done in order to find new therapeutic agents able to improve survival in these patients. New monoclonal antibodies such as cluster of differentiation (CD)19, CD20, CD22, and CD52 have been developed. The rationale 
for the use of monoclonal antibodies is that lymphoblasts express various cell-surface antigens that may be favourable targets for this therapy. For instance, over $95 \%$ of B-cell ALL and more than $90 \%$ of lymphoblasts express CD19 and CD22, respectively (32). Monoclonal antibody therapy has been recently used in clinical trials to treat children and adults with relapsed or refractory ALL. The initial results have been favourable with good tolerability and high levels of negative minimal residual disease. However, longer follow-up time is necessary to assess toxicity and long-term outcome.

\section{Acute myeloid leukaemia}

Similar to ALL, risk-adapted therapy has become critical for AML. AML has been risk stratified in two major groups. The low risk group (about $25 \%$ of the cases) includes patients with core-binding factor (CBF) AML [t(8;21), inv(16), $\mathrm{t}(15 ; 17)]$, infant AML, AML with Down syndrome, AML with CEBPA and NPM1 mutations (non-FLT3-ITD) or megakaryoblastic AML with the $\mathrm{t}(1 ; 22)$ abnormality, and minimal residual disease negative. The high-risk group (about $25 \%$ of the cases) includes patients with unfavourable cytogenetic alterations (monosomies 5 and 7), FLT3-ITD and TP53 mutations, secondary AML, AML associated with myelodisplastic syndrome, and minimal residual disease positive. In approximately $40-50 \%$ of cases there is not a good genetic or molecular marker to determine the disease prognosis and clinicians use minimal residual disease assessment to guide treatment (33). In general, the treatment of AML is performed using four to five intensive courses of cytarabine and anthracyclines chemotherapy. Maintenance therapy appears not to have any advantage in AML as it occurs for ALL. Central nervous system directed therapy with triple agents is also recommended for AML. Haematopoietic stem cell transplantation is performed more often among young patients with AML than for ALL (about 30 vs. 5\%).

\section{Core-binding factor AML}

CBF AML is associated with chromosomal rearrangements between chromosomes 8 and 21 (t8;21) and within chromosome 16 [inv(16)]. This subtype accounts for about $25 \%$ of all childhood AML cases and its prevalence decreases with advancing age. CBF AML prevalence is approximately $10-15 \%$ in adults aged 60 years or younger and $5 \%$ in patients older than 60 years (34). With intensive chemotherapy regimens with three to four drugs, CBF AML has become a group of good prognosis with 3-year overall survival of approximately $90 \%$ in children and adolescents and about $69 \%$ for young adults. Furthermore, haematopoietic stem cell tansplantation is not recommended for children in complete remission, but might be indicated for those who relapse.

\section{Down syndrome effects}

The patients diagnosed with both AML and Down syndrome have a better prognosis than non-Down syndrome patients with AML. Therefore, the current treatment approach is to reduce chemotherapy agents in order to avoid complications of treatment, particularly cardiotoxicity.

\section{Neonatal and infant acute myeloid leukaemia}

Because neonates with AML may have spontaneous remission, some clinicians may choose to observe them rather than begin chemotherapy immediately. When chemotherapy is necessary, careful dose adjustments should be done in order to avoid toxicity. Neonates tend to have worse clinical course due to complications of treatment and disease resistance than children. On the contrary, infants with AML often have similar prognosis as older children provided that they receive intensive chemotherapy regimen. Haematopoietic stem cell transplantation seems not to improve outcome in these patients and can cause serious adverse effects.

AML with altered genes (FLT3-ITD, NPM and CEBPA mutations) FLT3-ITD mutations are associated with poor prognosis in children (5-year overall survival <35\%) (35). The use of haematopoietic stem cell transplantation is still controversial, being usually reserved for high-risk patients. In contrast, patients with FTL3 point mutation have a better outcome and are often treated with chemotherapy only. Patients with NPM and CEBPA mutations have a favourable prognosis; therefore haematopoietic stem cell transplantation is not usually recommended for these patients. AML with MLL-rearrangements is a heterogeneous disease and prognosis may vary from $22 \%$ for patients with $\mathrm{t}(6 ; 11)$ to $100 \%$ for those with $\mathrm{t}(1 ; 11)$. The role of haematopoietic stem cell transplantation remains controversial in this subtype of disease (36).

\section{Conclusion}

The prognosis of acute leukaemia has improved substantially in the last few decades, mainly for ALL and some subtypes of AML. This improvement was possible due to national and international collaborative clinical trials that investigate the association of various factors on outcome. Risk-adapted therapy based on patient' clinical and genetic features and minimal residual disease assessment have largely contributed to treatment success in both ALL and AML treatments. However, for specific types of disease, prognosis is still poor and new treatment approaches are warranted.

\section{References}

1. Li Z, Zhang P, Yan A, Guo Z, Ban Y, Li J, Chen S, Yang H, He Y, Li J, et al: ASXL1 interacts with the cohesin complex to maintain chromatid separation and gene expression for normal hematopoiesis. Sci Adv 3: e1601602, 2017.

2. Szilvassy SJ: The biology of hematopoietic stem cells. Arch Med Res 34: 446-460, 2003.

3. Pui CH (ed): Acute lymphoblastic leukemia. In: Childhood Leukemias. 3rd edition. Cambridge University Press, Cambridge, pp332-366, 2012

4. Collins L, Beaumont L, Cranston A, Savoie S, Nayiager T and Barr R: Anthropometry in long-term survivors of acute lymphoblastic leukemia in childhood and adolescence. J Adolesc Young Adult Oncol: Jan 24, 2017 (Epub ahead of print).

5. El-Rifai W, Ruutu T, Elonen E, Volin L and Knuutila S: Prognostic value of metaphase-fluorescence in situ hybridization in follow-up of patients with acute myeloid leukemia in remission. Blood 89: 3330-3334, 1997.

6. Onciu M and Pui CH: Diagnosis and classification. In: Childhood Leukemias. Pui CH (ed). 3rd edition. Cambridge University Press, Cambridge, pp21-48, 2012.

7. Ferlay J, Soerjomataram I, Dikshit R, Eser S, Mathers C, Rebelo M, Parkin DM, Forman D and Bray F: Cancer incidence and mortality worldwide: sources, methods and major patterns in GLOBOCAN 2012. Int J Cancer 136: E359-E386, 2015. 
8. Rodriguez-Galindo C, Friedrich P, Morrissey L and Frazier L: Global challenges in pediatric oncology. Curr Opin Pediatr 25 3-15, 2013.

9. Bray F, Jemal A, Grey N, Ferlay J and Forman D: Global cancer transitions according to the Human Development Index (2008-2030): a population-based study. Lancet Oncol 13: 790-801, 2012.

10. Creutzig U, Büchner T, Sauerland MC, Zimmermann M, Reinhardt D, Döhner H and Schlenk RF: Significance of age in acute myeloid leukemia patients younger than 30 years: a common analysis of the pediatric trials AML-BFM 93/98 and the adult trials AMLCG 92/99 and AMLSG HD93/98A. Cancer 112: 562-571, 2008.

11. Deschler B and Lübbert M: Acute myeloid leukemia: epidemiology and etiology. Cancer 107: 2099-2107, 2006.

12. Pui CH, Robison LL and Look AT: Acute lymphoblastic leukaemia. Lancet 371: 1030-1043, 2008.

13. Pui CH: Acute lymphoblastic leukemia: introduction. Semin Hematol 46: 1-2, 2009.

14. Pui CH, Carroll WL, Meshinchi S and Arceci RJ: Biology, risk stratification, and therapy of pediatric acute leukemias: an update. J Clin Oncol 29: 551-565, 2011.

15. Pui CH, Mullighan CG, Evans WE and Relling MV: Pediatric acute lymphoblastic leukemia: where are we going and how do we get there? Blood 120: 1165-1174, 2012.

16. Goulden NJ, Knechtli CJ, Garland RJ, Langlands K, Hancock JP, Potter MN, Steward CG and Oakhill A: Minimal residual disease analysis for the prediction of relapse in children with standard-risk acute lymphoblastic leukaemia. Br J Haematol 100: 235-244, 1998

17. Veerman AJ, Kamps WA, van den Berg H, van den Berg E, Bökkerink JP, Bruin MC, van den Heuvel-Eibrink MM Korbijn CM, Korthof ET, van der Pal K, et al; Dutch Childhood Oncology Group: Dexamethasone-based therapy for childhood acute lymphoblastic leukaemia: results of the prospective Dutch Childhood Oncology Group (DCOG) protocol ALL-9 (1997-2004). Lancet Oncol 10: 957-966, 2009.

18. Schrappe M, Hunger SP, Pui CH, Saha V, Gaynon PS, Baruchel A, Conter V, Otten J, Ohara A, Versluys AB, et al: Outcomes after induction failure in childhood acute lymphoblastic leukemia. N Engl J Med 366: 1371-1381, 2012

19. Balduzzi A, Valsecchi MG, Uderzo C, De Lorenzo P, Klingebiel T, Peters C, Stary J, Felice MS, Magyarosy E, Conter V, et al: Chemotherapy versus allogeneic transplantation for very-highrisk childhood acute lymphoblastic leukaemia in first complete remission: comparison by genetic randomisation in an international prospective study. Lancet 366: 635-642, 2005.

20. Bleyer A, Budd T and Montello M: Adolescents and young adults with cancer: the scope of the problem and criticality of clinical trials. Cancer 107 (Suppl): 1645-1655, 2006.

21. Nachman J: Clinical characteristics, biologic features and outcome for young adult patients with acute lymphoblastic leukaemia. Br J Haematol 130: 166-173, 2005.

22. Bhojwani D, Pei D, Sandlund JT, Jeha S, Ribeiro RC, Rubnitz JE, Raimondi SC, Shurtleff S, Onciu M, Cheng C, et al: ETV6-RUNX1-positive childhood acute lymphoblastic leukemia: improved outcome with contemporary therapy. Leukemia 26: 265-270, 2012

23. Maloney KW, Carroll WL, Carroll AJ, Devidas M, Borowitz MJ, Martin PL, Pullen J, Whitlock JA, Willman CL, Winick NJ, et al: Down syndrome childhood acute lymphoblastic leukemia has a unique spectrum of sentinel cytogenetic lesions that influences treatment outcome: a report from the Children's Oncology Group. Blood 116: 1045-1050, 2010.
24. Hunger SP, Loh ML, Whitlock JA, Winick NJ, Carroll WL, Devidas M and Raetz EA; COG Acute Lymphoblastic Leukemia Committee: Children's Oncology Group's 2013 blueprint for research: acute lymphoblastic leukemia. Pediatr Blood Cancer 60: 957-963, 2013.

25. Nachman JB, La MK, Hunger SP, Heerema NA, Gaynon PS, Hastings C, Mattano LA Jr, Sather H, Devidas M, Freyer DR, et al: Young adults with acute lymphoblastic leukemia have an excellent outcome with chemotherapy alone and benefit from intensive postinduction treatment: a report from the Children's Oncology Group. J Clin Oncol 27: 5189-5194, 2009.

26. Stock W, Luger S, Advani A, Geyer S, Harvey R, Mullighan C, Willman CL, Malnassy G, Parker E, Laumann KM, et al: Favorable outcomes for older adolescents and young adults (AYA) with acute lymphoblastic leukemia (ALL): early results of US intergroup trial C10403. Blood 124: 796, 2014.

27. Larsen EC, Salzer W, Nachman J, Devidas M, Freyer DR, Raetz EA, Winick N, Hunger SP and Carroll WL: Treatment toxicity in adolescents and young adult (AYA) patients compared with younger patients treated for high risk B-precursor acute lymphoblastic leukemia (HR-ALL): a report from the Children's Oncology Group study AALL0232. Blood 118: 1510, 2011.

28. Carlson RH: Renewed calls for adolescents and young adults with ALL to be treated with pediatric protocols. Oncol Times 37: 20-21, 2015

29. Advani AS, Hunger SP and Burnett AK: Acute leukemia in adolescents and young adults. Semin Oncol 36: 213-226, 2009.

30. Gramatges MM and Rabin KR: The adolescent and young adult with cancer: state of the art - acute leukemias. Curr Oncol Rep 15: 317-324, 2013.

31. Nguyen K, Devidas M, Cheng SC, La M, Raetz EA, Carroll WL, Winick NJ, Hunger SP, Gaynon PS and Loh ML; Children's Oncology Group: Factors influencing survival after relapse from acute lymphoblastic leukemia: a Children's Oncology Group study. Leukemia 22: 2142-2150, 2008.

32. DeAngelo DJ: The use of novel monoclonal antibodies in the treatment of acute lymphoblastic leukemia. Hematology Am Soc Hematol Educ Program 2015: 400-405, 2015.

33. Rubnitz JE, Inaba H, Dahl G, Ribeiro RC, Bowman WP, Taub J, Pounds S, Razzouk BI, Lacayo NJ, Cao X, et al: Minimal residual disease-directed therapy for childhood acute myeloid leukaemia: results of the AML02 multicentre trial. Lancet Oncol 11: 543-552, 2010.

34. Mullighan C, Hunger SP and Meshinchi S: Molecular genetics in children, adolescents and young adults with acute lymphoblastic leukemia and acute myeloid leukemia. In: Hematological Malignancies in Children, Adolescents and Young Adults. Cairo MS and Perkins SL (eds). World Scientific Publishing Co. Pte. Ltd., Singapore, pp121-142, 2012.

35. Pratz KW, Sato T, Murphy KM, Stine A, Rajkhowa T and Levis M: FLT3-mutant allelic burden and clinical status are predictive of response to FLT3 inhibitors in AML. Blood 115: 1425-1432, 2010

36. Balgobind BV, Raimondi SC, Harbott J, Zimmermann M, Alonzo TA, Auvrignon A, Beverloo HB, Chang M, Creutzig U, Dworzak MN, et al: Novel prognostic subgroups in childhood 11q23/MLL-rearranged acute myeloid leukemia: results of an international retrospective study. Blood 114: 2489-2496, 2009. 